\title{
Décadrages Décadrages
}

cinéma, à travers champs Cinéma, à travers champs

40-42 | 2019

Cinéma ethnographique

\section{Éditorial}

François Bovier, Serge Margel, Achilleas Papakonstantis et Sylvain Portmann

\section{(2) OpenEdition}

Journals

Édition électronique

URL : https://journals.openedition.org/decadrages/1394

DOI : $10.4000 /$ decadrages. 1394

ISSN : 2297-5977

\section{Éditeur}

Association Décadrages

\section{Édition imprimée}

Date de publication : 1 novembre 2019

Pagination : 4-9

ISBN : 978-2-9700963-6-8

ISSN : 2235-7823

Référence électronique

François Bovier, Serge Margel, Achilleas Papakonstantis et Sylvain Portmann, «Éditorial », Décadrages [En ligne], 40-42 | 2019, mis en ligne le 01 septembre 2021, consulté le 04 avril 2022. URL : http:// journals.openedition.org/decadrages/1394; DOI : https://doi.org/10.4000/decadrages.1394 


\section{Éditorial}

CE dossier, CONSACRÉ AU CINÉmA ETHNOGRAPHIQUe, repose sur deux ensembles distincts mais néanmoins étroitement articulés: le film ethnographique tel qu'il s'est développé depuis les années i950 au sein de comités nationaux en France ou en Belgique (ceux-ci étant regroupés au sein d'un Comité du film ethnographique international) d'une part, et l'anthropologie visuelle telle qu'elle s'est déployée aux États-Unis, infléchissant le film ethnographique dans une direction résolument artistique, d'autre part. Le premier volet de ce dossier est connu de ce côté de l'Atlantique, voire résumé au travail de Jean Rouch, dont l'œuvre est encore trop souvent départagée entre documents destinés à des spécialistes et films adressés aux cinéphiles, alors que lui-même a toujours explicitement contesté cette distinction entre intention scientifique et visée artistique. Le second volet - il s'agit pourtant là de films produits et réalisés par des cinéastes-ethnographes très largement reconnus dans le champ de l'anthropologie visuelle - n'est guère pris en compte dans l'histoire et la théorisation du cinéma documentaire. En effet, dans l'espace francophone, le champ des études cinématographiques fait l'impasse sur les films de Tim Asch, Robert Gardner, John Marshall, pour citer les plus reconnus d'entre eux - sans parler des essais filmiques pionniers des anthropologues Gregory Bateson et Margaret Mead. Pourtant, The Hunters (John Marshall, 1957), Dead Birds (Robert Gardner, 1963), The Ax Fight (Tim Asch avec l'anthropologue Napoleon Chagnon, 1975) constituent des jalons incontournables pour qui veut étudier le cinéma ethnographique - ces films sont d'ailleurs régulièrement présentés dans les cursus universitaires liés à l'ethnographie et à l'anthropologie, l'Institut d'ethnologie de l'Université de Neuchâtel, pour prendre un exemple local, ayant acquis des copies $16 \mathrm{~mm}$ de certains de ces films qui sont par ailleurs tous diffusés par DER (Documentary Educational Resources).

Le présent dossier entend interroger, rapprocher et distinguer ces démarches qui émergent autour des années 1940-1950 et tendent à s’institutionnaliser dans les années 1960-I970. Dans ce but, nous avons fait appel à différents spécialistes, pour certains inscrits dans le champ des études cinématographiques, mais la plupart dans celui de l'anthro- 
pologie visuelle. Ce dossier porte donc sur des films qui font figure de «classiques» et qui ont fait l'objet d'analyses approfondies (à l'instar des Maîtres fous de Jean Rouch ou des Statues meurent aussi de Chris Marker et Alain Resnais), mais il aborde aussi et surtout des démarches filmiques, dont l'invention formelle et le goût pour l'expérimentation nous paraissent pourtant évidents, qui n’ont guère été intégrées à la théorisation du cinéma documentaire, du moins en dehors des États-Unis. Certes, comparaison n'est pas raison. Il faut distinguer l'histoire coloniale européenne qui surdétermine les conditions de réalisation de films ethnographiques adossés à des institutions scientifiques répondant à des missions scientifiques, culturelles, politiques déterminées, de la situation nord-américaine qui entretient un autre rapport à la colonisation. Et ce d'autant plus que les institutions qui soutiennent le cinéma ethnographique aux États-Unis sont souvent affiliées aux milieux artistiques (rappelons que le Film Study Center, fondé en I957 dans le contexte du Peabody Museum d'art et d'archéologie, est intégré au Carpenter Center for the Visual Arts en 1964).

Le dossier s'ouvre sur une contribution de Caroline Eades, spécialiste du cinéma postcolonial, qui interroge le fonctionnement institutionnel du Comité du film ethnographique (CFE) français, rattaché au Musée de l'Homme. Elle met en évidence l'exigence de rigueur scientifique et professionnelle des films de recherche qui répondent à l'idéal d'une bonne lisibilité des images, afin de garantir le large rayonnement de ceux-ci. Si le CFE vise à légitimer l'utilisation du cinéma dans une discipline scientifique relativement nouvelle et prône une professionnalisation et autonomie des ethnocinéastes, son action s’inscrit néanmoins dans le contexte d'un colonialisme de gauche qui reprend à son compte l'idée de «mission civilisatrice», comme l'expose avec pertinence la chercheuse. Deux articles reviennent sur le rôle central joué par Jean Rouch dans le développement du cinéma ethnographique dans les années i950, l'ethnocinéaste introduisant dans les protocoles jusqu'ici stricts de tournage une caméra participative et une propension à faire récit ou légende. Jamie Berthe, qui a soutenu une thèse de doctorat sur les liens de Jean Rouch à l'histoire coloniale française et au cinéma africain des indépendances, revient sur le premier film de Rouch qui rompt radicalement avec les conventions du cinéma ethnographique (voix over, stabilité de la caméra généralement installée sur un trépied, lisibilité des plans, 
visée didactique et approche scientifique du sujet), Bataille sur le grand fleuve (195I). Réinscrivant ce film dans la trajectoire de Rouch, Jamie Berthe souligne l'ambiguïté de son propos, tendant à l'émancipation de la culture des Nigériens sans dénoncer pour autant le contexte colonial duquel il ne parvient pas à s'extraire. La chercheuse pondère cependant cette critique en relevant la dynamique résolument collaborative des films de Rouch, qui peuvent ouvrir à d'autres approches de cultures lointaines. Jean-Paul Colleyn, quant à lui, documentariste et anthropologue, spécialiste du Mali, propose une lecture du film de Rouch Les Maîtres fous (I954). D’un côté, il montre comment ce film renverse les conventions du cinéma ethnographique et crée des passerelles entre science et art, entre écriture et cinéma, entre documentaire et fiction. De l'autre, il rattache la dimension politique du rituel de possession mis en scène dans le film à une volonté collective de contrôler des forces, ici coloniales, qui gouvernent la vie. Dans son étude sur Les Statues meurent aussi (1953) de Chris Marker et d'Alain Resnais, Matthias De Groof analyse l'une des premières critiques du colonialisme intentées par le biais du cinéma. Ce film s'est donné pour tâche, en effet, de critiquer le regard dominant sur les artefacts africains et le statut des objets muséographiques. La volonté d'exposer des objets non occidentaux, suivant la logique moderniste des musées, construit un certain primitivisme auquel la civilisation occidentale peut se référer comme à une variation du passé occidental qui perdure dans le présent. Damien Mottier consacre son article aux Gestes du repas (I958), essai d'ethno-fiction cinématographique de Luc de Heusch, qui capte les gestes quotidiens des Belges en train de manger. Son hypothèse porte sur un déplacement dans le fonctionnement institutionnel du film ethnographique. Il montre comment le film de Luc de Heusch, qui a d'abord été conçu en vue de renforcer la coopération internationale, révèle un certain repli franco-belge du Comité international du film ethnographique. Par ce fait, il annonce la reprise des enjeux théoriques et épistémologiques de l'ethnographie européenne par l'anthropologie américaine, qui ouvrira le champ dès les années i96o à l'anthropologie visuelle.

Le second volet de ce dossier, nous l'avons dit, porte sur le cinéma ethnographique américain; les auteurs sollicités développent tous une perspective monographique. Dans son article, Moira Sullivan aborde les relations de travail entre Maya Deren, Margaret Mead et Gregory 
Bateson, en prenant comme point de départ le projet filmique de i936I937 de Mead et Bateson à Bali. Elle soutient deux hypothèses principales. D’une part, le film de Mead et Bateson consiste à filmer des séquences d'actions en observant certains comportements selon les règles de l'objectivité: la caméra est frontale, distante du sujet, les plans sont longuement tenus, sans mouvements de caméra. De l'autre, elle montre que, pour Maya Deren, la tâche de la cinéaste est semblable à celle d'une chorégraphe: les prises de vue de Deren se concentrent sur des danses en l'honneur d'une divinité, qu'elle filme en tant qu'observatriceparticipante. Le texte de William Rothman sur les films de Robert Gardner se concentre sur les années 1950-1960. Il montre que cette œuvre repose sur une certaine tension, le cinéaste dirigeant simultanément son regard sur les autres et sur lui-même. Les différentes sociétés qu'il filme sont abordées tant dans leur altérité que comme un miroir de notre propre société. Rothman montre combien Gardner voulait que son œuvre soit poétique, en articulant la littérature et l'anthropologie, l'art et la science. Dans une étude approfondie, Carolyn Anderson retrace le parcours filmique de John Marshall, en montrant comment le cinéaste anticipe un tournant dans le cinéma documentaire et ethnographique, en interrogeant les relations entre l'observateur et le sujet observé. Elle insiste tout particulièrement sur ses courts «films-séquences» dans les années i960, qui consistent en de longues prises de vues, se réduisant parfois même à un seul plan. Cette forme filmique implique une dimension réflexive, en ne cherchant pas à masquer les interventions du cinéaste au moment du tournage. L'article de Nancy Lutkehaus se concentre sur la période de formation de Tim Asch durant les années i950 et I960, soit avant sa série de films au Venezuela sur les Yanomami, pour laquelle il est principalement reconnu. Elle montre comment Asch, photographe de formation, est devenu réalisateur de films ethnographiques, à une époque où la relation entre le cinéma et l'ethnographie n'était pas encore reconnue et où l'anthropologie était toujours à la recherche d'une légitimité académique. Dans son entretien avec Scott MacDonald, Craig Johnson retrace l'aventure que représentaient les films de Tim Asch et de l'anthropologue Napoleon Chagnon sur les Yanomami. Craig Johnson, désigné par MacDonald dans son entretien comme le «troisième homme», fut entre autres le preneur de son et le monteur des films de la série Yanomami. La première partie de l'entretien est consacrée à 
la présentation des films de Asch et à sa collaboration avec Chagnon. La seconde partie consiste en une discussion sur la contribution de Craig Johnson aux films sur les Yanomami, en particulier The Ax Fight, en l'inscrivant dans le contexte plus large de son initiation au cinéma. Pour leur part, François Bovier et Serge Margel montrent comment on peut comprendre les liens complexes entre ethnographie et cinéma au travers de formes spécifiques et expérimentales, comme la pratique du «film-séquence» qui se développe dès les années i960. La structure du film-séquence est liée aux notions d'action, d'événement, d'interaction et d'environnement - ce qui résonne avec la théorisation des events et des happenings dans le contexte du nouveau théâtre et du renouvellement des scènes artistiques. En tout cas, une tension s'établit dans le cinéma ethnographique des années I950-1960 entre le travail de mise en forme du film et la théâtralité de l'événement.

Ouvrant la rubrique suisse tout en prolongeant ce dossier sur le cinéma ethnographique, Francis Mobio revient sur Mademoiselle Rose de Pinsec (1978), un film de Jacques Thévoz. Ce dernier a été photographe et opérateur à la télévision. Dès les années i960, il inaugure une série de portraits, d'hommes et de femmes, la plupart en marge. Analysant le film documentaire sur Rose Monnet dans le village valaisan de Pinsec, Francis Mobio s’interroge sur les intentions de Thévoz. A-t-il voulu sauvegarder un patrimoine avant sa destruction ou sa mutation, comme on le dit le plus souvent? Mobio évoque au contraire un «pacte filmique» contracté entre Thévoz et Rose Monnet, qui ne consiste pas tant à sauver le passé qu'à tenter de relier ce qui est à ce qui a été, c'està-dire les anciens de Pinsec aux modernes nés en bas dans la vallée, dans les villes.

La suite de la rubrique suisse de ce numéro propose plusieurs analyses de films sortis récemment, ainsi que deux comptes rendus d'ouvrages sur le cinéma. Le premier article, signé Faye Corthésy, combine l'analyse en détail et le compte rendu: à l'occasion des vingt ans de Videoex, festival zurichois de cinéma expérimental, elle analyse une performance (la séance de Caspar Stracke intitulée What You Imagined It to Be) et le film Spell Reel de l'artiste portugaise Filipa César qui y ont été programmés. C’est le rapport de ces objets au temps et à l'histoire qui a retenu l'attention de la chercheuse, celle-ci approchant ces œuvres par le biais des liens qu'elles entretiennent avec les archives, opérant une dis- 
tinction entre nostalgie pour le passé et réalité politique de la mémoire - les questions soulevées par ces films n'étant pas sans échos avec les enjeux du cinéma ethnographique. Oscillant entre analyse esthétique et lecture herméneutique, Laure Cordonier traite du film Fortuna, deuxième long métrage de fiction du cinéaste suisse Germinal Roaux. En se penchant de plus près sur une séquence cruciale dans le déploiement du récit, Cordonier éclaire les implications de l'organisation formelle et stylistique du film - y compris la performance des acteurs - sur le discours véhiculé par ce dernier. Son article se clôt par un entretien avec Roaux qui relate son expérience en matière de réalisation filmique et revient sur sa collaboration avec le célèbre comédien suisse Bruno Ganz, décédé récemment. L'article d’Adèle Morerod envisage trois productions helvétiques récentes, Les Voyages extraordinaires d'Ella Maillart, Bis ans Ende der Traüme et Yvette Z'Graggen: une femme au volant de sa vie, à l'aune de leur réception critique dans la presse romande. Morerod articule l'analyse détaillée des discours avec celle des films afin d'interroger la catégorie de «docufiction», les connotations qui lui sont associées et les problématiques qui en découlent.

Roland Cosandey livre ici un compte rendu détaillé de l'ouvrage d'Olivier Robert, Petits théâtres lausannois de la Belle Époque, publié en 2015 aux Éditions d'en bas, à Lausanne. Il prolonge ainsi une série de contributions débutée il y a quelques années déjà dans la revue, dont le thème est les salles de cinéma en Suisse. Il relève la richesse des illustrations du livre tout en pondérant les propos de l'auteur à travers un certain nombre de réserves, qu'il articule précisément. Isabel Krek signe quant à elle une recension consacrée à une parution récente: Bewegte Bilder für eine entwickelte Welt. Die Dokumentarfilme von René Gardi, Ulrich Schweizer und Peter von Gunten in der Schweizer Entwicklungsdebatte, I959-I986 de Felix Rauh, qui porte sur l'impact du film documentaire sur le débat autour du développement en Suisse et, plus précisément, sur l'œuvre de trois cinéastes, René Gardi, Ulrich Schweizer et Peter von Gunten. L’analyse critique qu'elle livre de cet ouvrage lui permet de discuter les enjeux méthodologiques relatifs à l'étude à la fois esthétique et historico-politique de films documentaires dont la visée est humanitaire.

(fb, sm, ap, sp)

\section{Vernissage}

Institut d'ethnologie

Rue de Saint-Nicolas 4

$\mathrm{CH}-2000$ Neuchâtel

Dimanche 27 octobre, $13 h-17 h$

Projection de films $16 \mathrm{~mm}$ :

- The Wasp Nest (1972, 20'), John Marshall

- Weeding the Garden (1971, 14'), Timothy Asch, Napoleon A. Chagnon

- Forest of Bliss (1988, 90'), Robert Gardner 